\title{
Phytoplankton of two rivers in the eastern Amazon: characterization of biodiversity and new occurrences ${ }^{1}$
}

Elane Domênica de Souza Cunha ${ }^{2,4}$, Alan Cavalcanti da Cunha², Arialdo Martins da Silveira Jr. ${ }^{3}$ and Silvia Maria Mathes Faustino ${ }^{3}$

Submitted: 6 August, 2012. Accepted: 4 February, 2013

\begin{abstract}
During the various hydrological periods in 2011, we studied the phytoplankton along an 87-km stretch of the Araguari and Falsino rivers, which are located in the eastern Brazilian Amazon, in the state of Amapá, Brazil, the most ecologically preserved state in the country. In the study area, the aquatic ecosystem is under pressure from human activities such as, mining, hydroelectric power generation and urbanization, which contrast with the surrounding areas that are designated for biodiversity conservation. The aim of this study was to characterize the composition, frequency and richness of algae species and also to identify spatial-temporal patterns of taxa distribution. During the study period, we identified 185 taxa (136 species in 49 genera). The division Chlorophyta (class: Zygnematophyceae) presented the greatest number of taxa, whereas the division Rhodophyta accounted for only $1 \%$ of the taxa recorded. Most of the species identified (69\%) were classified as sporadic in occurrence. The few taxa that were classified as common belonged mainly to the group Bacillariophyta. Species richness was greatest richness in the lentic stretches (in a reservoir) and in November (during the dry season). Of the 185 taxa identified, 174 are new records for the state of Amapá.
\end{abstract}

Key words: algae, Amapá, floristic survey, reservoir, river

\section{Introduction}

Phytoplankton is composed of microscopic organisms, also known as algae, which remain at the surface of water bodies. These organisms provide essential environmental services such as oxygen production for the aquatic environment (Wehr \& Descy 1998) and for the atmosphere, from which they also sequester carbon dioxide (Ohse et al. 2009). In addition, these organisms form the base of the food chain of aquatic ecosystems and supply much of the carbon dioxide that is necessary to fishes, which are a primary source of protein for human populations (Araújo-Lima et al. 1986).

The Brazilian continental waters are rich in phytoplankton and present considerable phytoplankton biodiversity, with approximately $25 \%$ of the species in the world (Agostinho et al. 2005). Knowledge of the biodiversity of these algae is, however, quite heterogeneous regarding geographic region, taxonomic group (Bicudo \& Menezes 2010) and type of aquatic ecosystem (Soares et al. 2007).

In Brazil, data related to algae is generated primarily in the southern and southeastern regions of the country, followed by the northeastern region, where substantial knowledge of these organisms has been gathered, despite the scarcity of hydrological resources in the region. Very little is known regarding the biodiversity of algae in the central-west and northern regions of Brazil, where the largest watershed in the world is located (Bicudo \& Menezes 2010). Specifically in the state of Amapá, knowledge of phytoplankton is even scarcer, only two studies having been conducted to date (Förster 1964; Souza \& Melo 2011).

Certain taxonomic groups of phytoplankton have been more widely studied than others. This has been the case for the Amazon region, where few studies have addressed the phytoplankton community as a whole (Huszar \& Reynolds 1997; Ibañez 1998; Melo \& Huszar 2000; Melo et al. 2005a; Paiva et al. 2006; Aprile \& Mera 2007; Monteiro et al. 2009; Pereira et al. 2010; Almeida \& Melo 2011). Most studies conducted in the region have examined specific taxonomic groups, such as desmids (Grönblad 1945; Förster 1963, 1964, 1969, 1974; Scott et al. 1965; Thomasson 1971; Sophia \& Huszar 1996; Lopes \& Bicudo 2003; Melo et al. 2005b; Souza et al. 2007; Melo \& Souza 2009; Melo et al. 2009; Souza \& Melo 2011) and diatoms (Diaz-Castro et al. 2003; Ferrari et al. 2007; Pereira et al. 2012; Raupp et al. 2009;

\footnotetext{
${ }^{1}$ Based on the Master's dissertation of the first Author

${ }^{2}$ Universidade Federal do Amapá, Programa de Pós-Graduação em Biodiversidade Tropical, Macapá, AP, Brazil

${ }^{3}$ Universidade Federal do Amapá, Programa de Pós-Graduação em Ciências da Saúde, Macapá, AP, Brazil

${ }^{4}$ Author for correspondence: elanedsc@yahoo.com.br
} 
Sala et al. 2002; Tudesque et al. 2012; Wetzel et al. 2012).

Most studies of algae in Brazil have involved small aquatic ecosystems, such as lakes, lagoons and reservoirs (Silva et al. 2001; Bozelli \& Huszar 2003). The phytoplankton biodiversity in the rivers of Brazil is still poorly studied, despite the extensive river system in the country (Soares et al. 2007). This is attributable, in part, to the high cost and complex logistics of conducting research on rivers in comparison with those of studies carried out in discrete environments (small lakes and reservoirs), features that Bozelli \& Huszar (2003) referred to as "methodological difficulties".

In this context, it is necessary to increase research efforts regarding phytoplankton biodiversity, especially in the Amazon, where there are plans for the construction of hydroelectric plants, which significantly alter the flow of rivers and, consequently, the dynamics of aquatic organisms (Zeng et al. 2006). In addition, this is one the least served regions of Brazil regarding sewage treatment, as noted by Cunha et al. (2004; 2005) and Agostinho et al. (2005). According to those authors, the construction of dams in the region will compound the problems caused by inadequate sewage treatment. Taken separately or together, these two factors present a major threat to aquatic biodiversity, potentially resulting in local extinctions and alterations within communities.

In the region of our study site, the aquatic ecosystem is facing pressures caused by human activities such as mining, construction of medium-sized hydroelectric plants, and urbanization, activities that put the site in contrast with the surrounding areas, which are dedicated to biodiversity conservation. In addition to a recently constructed hydroelectric plant, another two hydroelectric plants are currently under construction on the same stretch of river (Cunha et al. 2011; 2013). In this scenario, the objective of the present study was to record the biodiversity within this ecosystem prior to its further modification. By characterizing the composition, frequency and richness of the phytoplankton communities in two Amazonian rivers (one in a conservation area and one already under the influence of hydroelectric plants), identifying spatial and temporal patterns in the distribution of taxa, we hope to improve the knowledge of phytoplankton biodiversity in such rivers.

\section{Material and methods}

The state of Amapá is located in the extreme north of Brazil, in the eastern portion of the Amazon basin, and $62 \%$ of its territory is set aside through the establishment of constructs such as conservation units, environmentally protected areas, legal reserves, remaining territories of quilombos (Brazilian settlements founded by escaped or freed slaves) and lands reserved for use by indigenous tribes, making it the most ecologically preserved state in the country (Brito, 2008).

The Araguari and Falsino rivers are part of the watershed of the Araguari river, the largest watershed exclusive to the state of Amapá. These are considered "clear-water" rivers, because of the small quantity of suspended particles (Junk et al. 2012), are acidic (like the majority of Amazonian rivers) and contain $6-8 \mathrm{mg} / \mathrm{L}$ of dissolved oxygen (Bárbara et al. 2010; Cunha et al. 2011). According to the Brazilian National Environmental Council, these rivers are categorized as class II, meaning that their water is potable (after conventional treatment); their aquatic species are protected; and they are open to recreation, irrigation, aquiculture and fishing (Brasil, 2005). These rivers are turbulent, because the declivity is $0.50 \%$ at the beginning of the stretch (site F1), resulting in high flow rates, which decrease near the Coaracy Nunes Hydroelectric Plant (CNHP) reservoir, where the declivity drops to $0.35 \%$, and are considered to have a high self-purification capacity (Bárbara et al. 2010; Cunha et al. 2011; 2013).

The stretch of river selected for this study is $87 \mathrm{~km}$ long, beginning at the Amapá National Forest, on the Falsino river, and ending at the CNHP reservoir on the Araguari river (Fig. 1). This stretch is strategically important regarding land use, which varies from conservation units to human activities that are common in the Amazon basin, such as mining, including pebble extraction (Bárbara et al. 2010); urbanization (small- to medium-sized cities); and, mainly, the generation of hydroelectric energy (Cunha et al. 2011).

We conducted phytoplankton surveys at 14 sampling sites arranged as indicated in Tab. 1. These sites-designated F1 (within a conservation unit on the Falsino river); P1-P4 and P6-P9 (on lands used for various purposes along both rivers); and R1-R5 (on the CNHP reservoir, Araguari river)-were chosen in order to continue the historic water quality series developed by Bárbara et al. (2010) and Cunha et al. (2010) on the Araguari and Falsino rivers. Our surveys were conducted in February, May, August and November of 2011, corresponding to the following hydrological periods, respectively: dry-wet seasonal transition, wet season, wetdry seasonal transition and dry season.

We obtained phytoplankton samples using a $20 \mu \mathrm{m}$ mesh plankton net. A total of 56 samples were collected. The samples were subsequently fixed in Transeau's solution (Bicudo \& Menezes 2006). The taxa were identified under standard light microscopy with the aid of specialized bibliography, which included but was not limited to Grönblad (1945), Förster (1963; 1964), Komárek \& Anagnostidis (1999; 2005) and Bicudo \& Menezes (2006). Whenever possible, specimens were identified down to the species level.

We used the system devised by Hoek et al. (1995) to arrange the taxa in divisions and classes, except for the division Heterokontophyta, which was separated into Bacillariophyta and Chrysophyta. This procedure was adopted to facilitate the comparison with similar studies, because most still make use of this nomenclature. The classification of diatoms followed Medlin \& Kaczmarska (2004).

The frequency for each taxon (expressed as a percentage) was calculated with the following formula:

(number of sample sites $\times 100) / 56$ 


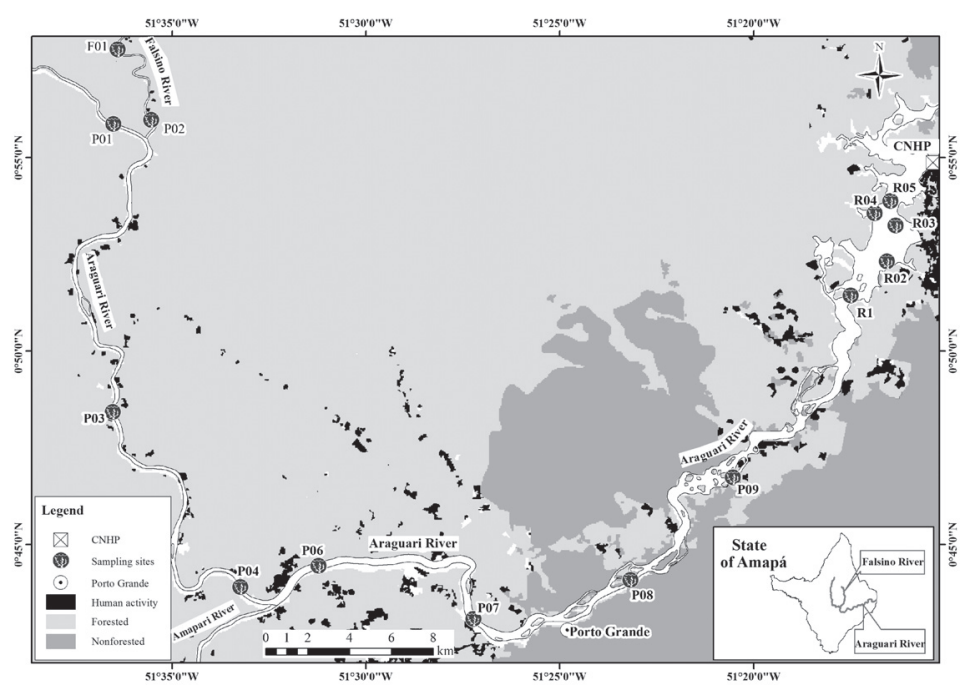

Figure 1. Map of the study site. Sampling sites on the Araguari and Falsino rivers.

Table 1. Description of the sampling sites on the Araguari and Falsino rivers.

\begin{tabular}{|c|c|c|c|}
\hline Site & Location, characteristics and land use & \multicolumn{2}{|c|}{ Geographic coordinates } \\
\hline $\mathrm{F} 1$ & (F) Conservation unit, Amapá National Forest & $0^{\circ} 57^{\prime} 37.00^{\prime \prime} \mathrm{N}$ & $51^{\circ} 36^{\prime} 22.00^{\prime \prime} \mathrm{W}$ \\
\hline $\mathrm{P} 1$ & (A) Conservation unit, Amapá National Forest & $0^{\circ} 55^{\prime} 47.70^{\prime \prime} \mathrm{N}$ & $51^{\circ} 36^{\prime} 33.48^{\prime \prime} \mathrm{W}$ \\
\hline $\mathrm{P} 2$ & (F) Conservation unit, Amapá National Forest & $0^{\circ} 55^{\prime} 54.48 ” \mathrm{~N}$ & $51^{\circ} 35^{\prime} 37.20^{\prime \prime} \mathrm{W}$ \\
\hline P3 & (A) Conservation unit, Amapá State Forest & $0^{\circ} 48^{\prime} 24.00^{\prime \prime} \mathrm{N}$ & $51^{\circ} 36^{\prime} 37.80^{\prime \prime} \mathrm{W}$ \\
\hline $\mathrm{P} 4$ & (A) Upstream of the confluence with the Amapari river & $0^{\circ} 43^{\prime} 51.96 ” \mathrm{~N}$ & $51^{\circ} 33^{\prime} 21.54^{\prime \prime} \mathrm{W}$ \\
\hline P6 & (A) Downstream of the confluence with the Amapari river; mining & $0^{\circ} 44^{\prime} 12.18^{\prime \prime} \mathrm{N}$ & $51^{\circ} 31^{\prime} 12.48^{\prime \prime} \mathrm{W}$ \\
\hline $\mathrm{P} 7$ & (A) Municipality of Porto Grande; urbanization, mining, extraction of pebbles and ornamental stones & $0^{\circ} 43^{\prime} 03.70^{\prime \prime} \mathrm{N}$ & $51^{\circ} 27^{\prime} 15.40^{\prime \prime} \mathrm{W}$ \\
\hline P8 & (A) Downstream of the municipality of Porto Grande; mining & $0^{\circ} 44^{\prime} 02.88^{\prime \prime} \mathrm{N}$ & $51^{\circ} 23^{\prime} 07.50^{\prime \prime} \mathrm{W}$ \\
\hline P9 & (A) Downstream of waterfalls, river-reservoir transition & $0^{\circ} 46^{\prime} 47.40^{\prime \prime} \mathrm{N}$ & $51^{\circ} 20^{\prime} 30.96^{\prime \prime} \mathrm{W}$ \\
\hline $\mathrm{R} 1$ & (A) Reservoir (Coaracy Nunes Hydroelectric Plant) & $0^{\circ} 51^{\prime} 11.88^{\prime \prime} \mathrm{N}$ & $51^{\circ} 17^{\prime} 22.92^{\prime \prime} \mathrm{W}$ \\
\hline $\mathrm{R} 2$ & (A) Reservoir (Coaracy Nunes Hydroelectric Plant) & $0^{\circ} 52^{\prime} 17.46^{\prime \prime} \mathrm{N}$ & $51^{\circ} 16^{\prime} 35.34^{\prime \prime} \mathrm{W}$ \\
\hline R3 & (A) Reservoir (Coaracy Nunes Hydroelectric Plant) & $0^{\circ} 53^{\prime} 13.20^{\prime \prime} \mathrm{N}$ & $51^{\circ} 16^{\prime} 21.78^{\prime \prime} \mathrm{W}$ \\
\hline $\mathrm{R} 4$ & (A) Reservoir (Coaracy Nunes Hydroelectric Plant) & $0^{\circ} 53^{\prime} 36.72^{\prime \prime} \mathrm{N}$ & $51^{\circ} 17^{\prime} 00.60^{\prime \prime} \mathrm{W}$ \\
\hline $\mathrm{R} 5$ & (A) Reservoir (Coaracy Nunes Hydroelectric Plant) & $0^{\circ} 53^{\prime} 53.16^{\prime \prime} \mathrm{N}$ & $51^{\circ} 16^{\prime} 35.94^{\prime \prime} \mathrm{W}$ \\
\hline
\end{tabular}

(F) - Falsino river; (A) - Araguari river.

where 56 is the total number of samples in the temporal and spatial axes. Taxa were then divided into the following frequency classes, adapted from Mateucci \& Colma (1982): quite common (> 70\%); relatively common $(70-40 \%)$; relatively uncommon $(40-10 \%)$; and sporadic $(\leq 10 \%)$.

To determine whether phytoplankton composition was similar among sampling sites, we conducted a cluster analysis with Ward's method and determined Euclidean distances using the software R-Project 2.14 (R Development Core Team 2012).

To verify new records for the state of Amapá, we reviewed studies on continental microalgae carried out in the state (Förster 1964; Souza \& Melo 2011).

The samples were deposited in the liquid collection of the herbarium of the Institute for Scientific and Technologi- cal Research in the State of Amapá (code, HAMAB) under the accession numbers 0000001 through 0000056.

\section{Results}

We recorded 185 taxa (Tab. 2) -49 identified down to the genus level and 136 identified down to the species level-within five divisions and ten classes. The divisions that were the most well-represented were Chlorophyta (with $71.9 \%$ of the taxa), Cyanophyta (with $14.6 \%$ ) and Bacillariophyta (with 10.9\%), Chrysophyta and Rhodophyta accounting for only $1.6 \%$ and $1.0 \%$, respectively (Fig. 2). 
Table 2. Complete list of taxa with their respective frequency classes and site of occurrence ( $\mathrm{n}$ of samples $=56$ ).

\section{DIVISION}

Class

Genus and species
FC

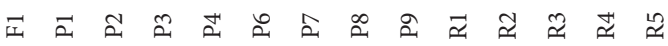

\section{CYANOPHYTA}

Cyanophyceae

Aphanocapsa delicatissima West \& West*

Aphanocapsa sp.*

Aphanothece sp.*

Aphanothece stagnina (Sprengel) Braun*

Bacularia gracilis Komárek*

Chamaesiphon sp.*

Chroococcus dispersus (Keissler) Lemmermann*

Cyanosarcina sp. ${ }^{*}$

Leptolyngbya sp.*

Lyngbya sp.*

Nostoc sp.*

Oscillatoria limosa Agardh ex Gomont*

Oscillatoria princeps Vaucher ex Gomont*

Phormidium autumnale (Agardh) Trevisan ex Gomont*

Phormidium granulatum (Gardner) Anagnostidis*

Phormidium retzii (Agardh) Gomont ex Gomont*

Phormidium sp.*

Phormidium stagninum (Kützing ex Gomont) Anagnostidis*

Phormidium tergestinum (Gomont) Anagnostidis \& Komárek*

Planktothrix agardhii (Gomont) Anagnostidis \& Komárek ${ }^{\star}$

Planktothrix isothrix (Skuja) Komárek \& Komárková*

Planktothrix sp.*

Pseudanabaena galeata Böcher*

Pseudanabaena mucicola (Naumann \& Huber-Pestalozzi) Schwabe*

Pseudanabaena sp.*

Scytonema sp.*

Stigonema sp.*

\section{RODOPHYTA}

Florideophyceae

Balliopsis prieurii (Kützing) Saunders \& Necchi-Júnior*

Batrachospermum sp.*

\section{BACILLARIOPHYTA**}

Bacillariophyceae

Actinella mirabilis Grunow*

Amphora delphinea Cleve*

Asterionella formosa Hassall ${ }^{\star}$

Eunotia pseudoserra De Oliveira \& Steinitz-Kannan*

Eunotia subrobusta Hustedt ${ }^{*}$
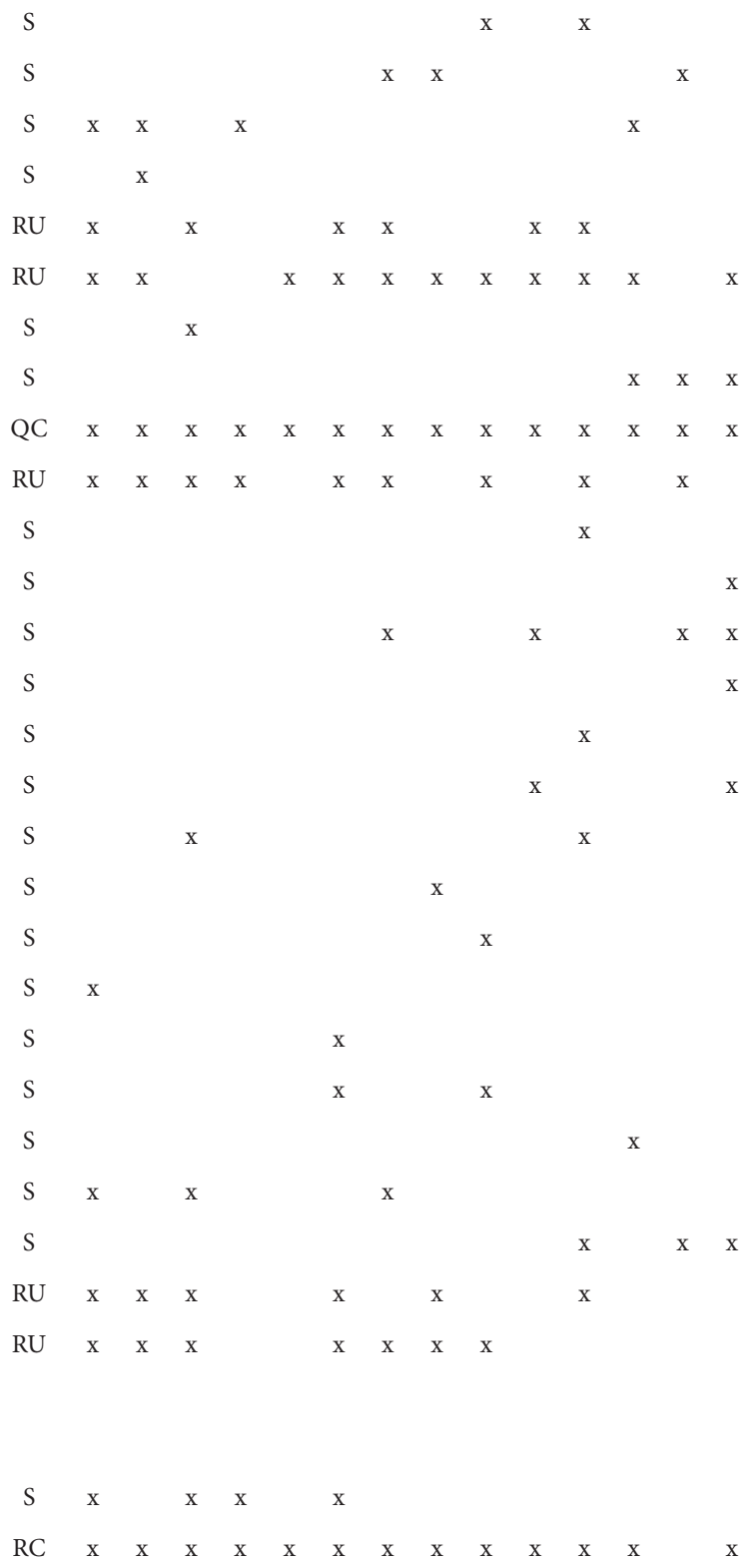

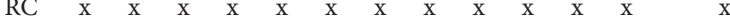


Table 2. Continuation.

\begin{tabular}{|c|c|c|c|c|c|c|c|c|c|c|c|c|c|c|c|}
\hline \multirow{2}{*}{$\begin{array}{l}\text { DIVISION } \\
\text { Class } \\
\text { Genus and species }\end{array}$} & \multirow{2}{*}{$\mathrm{FC}$} & \multicolumn{14}{|c|}{ Sampling sites } \\
\hline & & $\overrightarrow{\text { 死 }}$ & $\vec{a}$ & $\tilde{n}$ & $\tilde{2}$ & $\ddot{2}$ & $\stackrel{\circ}{2}$ & $\hat{\mathrm{A}}$ & $\stackrel{\infty}{\infty}$ & 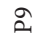 & $\vec{\simeq}$ & $\approx$ & $\cong$ & $\ddot{\sim}$ & $\mathscr{2}$ \\
\hline Eunotia yanomami Metzeltin \& Lange-Bertalot ${ }^{*}$ & RC & $\mathrm{x}$ & $\mathrm{x}$ & $\mathrm{x}$ & $\mathrm{x}$ & $\mathrm{x}$ & & $\mathrm{x}$ & $\mathrm{x}$ & $\mathrm{x}$ & $\mathrm{x}$ & $\mathrm{x}$ & $\mathrm{x}$ & $\mathrm{x}$ & $\mathrm{x}$ \\
\hline Fragilaria sp.* & RC & $\mathrm{x}$ & $\mathrm{x}$ & $\mathrm{x}$ & $\mathrm{x}$ & $\mathrm{x}$ & $\mathrm{x}$ & $\mathrm{x}$ & $\mathrm{x}$ & $\mathrm{x}$ & $\mathrm{x}$ & $\mathrm{x}$ & $\mathrm{x}$ & $\mathrm{x}$ & $\mathrm{x}$ \\
\hline Gomphonema neonasutum Lange-Bertalot \& Reichardt* & $S$ & & & & & & $\mathrm{x}$ & $\mathrm{x}$ & & & & & $\mathrm{x}$ & & \\
\hline Pinnularia sp.* & RU & $\mathrm{x}$ & $\mathrm{x}$ & $\mathrm{x}$ & $\mathrm{x}$ & $\mathrm{x}$ & & $\mathrm{x}$ & $\mathrm{x}$ & & & $\mathrm{x}$ & $\mathrm{x}$ & $\mathrm{x}$ & $\mathrm{x}$ \\
\hline Placoneis sp.* & S & & & $\mathrm{x}$ & & & $\mathrm{x}$ & & & $\mathrm{x}$ & & & $\mathrm{x}$ & & $\mathrm{x}$ \\
\hline Platessa sp.* & S & & & $\mathrm{x}$ & & & & & & & & & & & \\
\hline Stauroneis sp.* & $\mathrm{RU}$ & & & $\mathrm{x}$ & $\mathrm{x}$ & $\mathrm{x}$ & & $\mathrm{x}$ & & & $\mathrm{x}$ & $\mathrm{x}$ & & & $\mathrm{x}$ \\
\hline Surirella grunowii sensu Kulikovskiy, Lange-Bertalot \& Witkowski* & $\mathrm{RC}$ & $\mathrm{x}$ & $\mathrm{x}$ & $\mathrm{x}$ & $\mathrm{x}$ & $\mathrm{x}$ & $\mathrm{x}$ & $\mathrm{x}$ & $\mathrm{x}$ & $\mathrm{x}$ & $\mathrm{x}$ & $\mathrm{x}$ & $\mathrm{x}$ & $\mathrm{x}$ & $\mathrm{x}$ \\
\hline Surirella guatimalensis Ehrenberg* & RU & & & & $\mathrm{x}$ & & & & $\mathrm{x}$ & $\mathrm{x}$ & $\mathrm{x}$ & & $\mathrm{x}$ & & $\mathrm{x}$ \\
\hline Surirella linearis Smith* & QC & $\mathrm{x}$ & $\mathrm{x}$ & $\mathrm{x}$ & $\mathrm{x}$ & $\mathrm{x}$ & $\mathrm{x}$ & $\mathrm{x}$ & $\mathrm{x}$ & $\mathrm{x}$ & $\mathrm{x}$ & $\mathrm{x}$ & $\mathrm{x}$ & $\mathrm{x}$ & $\mathrm{x}$ \\
\hline Synedra sp.* & $S$ & & & & & $\mathrm{x}$ & & & & & & & $\mathrm{x}$ & & \\
\hline Tabellaria sp.* & $\mathrm{RC}$ & $\mathrm{x}$ & & $\mathrm{x}$ & $\mathrm{x}$ & $\mathrm{x}$ & $\mathrm{x}$ & $\mathrm{x}$ & $\mathrm{x}$ & $\mathrm{x}$ & $\mathrm{x}$ & $\mathrm{x}$ & $\mathrm{x}$ & $\mathrm{x}$ & $\mathrm{x}$ \\
\hline \multicolumn{16}{|l|}{ Coscinodiscophyceae } \\
\hline Aulacoseira granulata (Ehrenberg) Simonsen* & S & & & & & & & & & & $\mathrm{x}$ & $\mathrm{x}$ & & $\mathrm{x}$ & $\mathrm{x}$ \\
\hline Aulacoseira sp. ${ }^{*}$ & RU & $\mathrm{x}$ & $\mathrm{x}$ & $\mathrm{x}$ & & & & $\mathrm{x}$ & & & & & $\mathrm{x}$ & $\mathrm{x}$ & $\mathrm{x}$ \\
\hline \multicolumn{16}{|l|}{ Mediophyceae } \\
\hline Terpsinoe musica Ehrenberg* & $\mathrm{S}$ & & & & & & & & & $\mathrm{x}$ & $\mathrm{x}$ & & & & $\mathrm{x}$ \\
\hline \multicolumn{16}{|l|}{ CHRYSOPHYTA $^{* *}$} \\
\hline \multicolumn{16}{|l|}{ Chrysophyceae } \\
\hline Dinobryon sp.* & RU & & & $\mathrm{x}$ & & & & & & & $\mathrm{x}$ & $\mathrm{x}$ & $\mathrm{x}$ & $\mathrm{x}$ & $\mathrm{x}$ \\
\hline Rhipidodendron huxleyi Kent* & QC & $\mathrm{x}$ & $\mathrm{x}$ & $\mathrm{x}$ & $\mathrm{x}$ & $\mathrm{x}$ & $\mathrm{x}$ & $\mathrm{x}$ & $\mathrm{x}$ & $\mathrm{x}$ & $\mathrm{x}$ & $\mathrm{x}$ & $\mathrm{x}$ & $\mathrm{x}$ & $\mathrm{x}$ \\
\hline Synura uvella Ehrenberg* & RU & $\mathrm{x}$ & $\mathrm{x}$ & $\mathrm{x}$ & $\mathrm{x}$ & $\mathrm{x}$ & & $\mathrm{x}$ & $\mathrm{x}$ & $\mathrm{x}$ & $\mathrm{x}$ & $\mathrm{x}$ & $\mathrm{x}$ & $\mathrm{x}$ & $\mathrm{x}$ \\
\hline \multicolumn{16}{|l|}{ CHLOROPHYTA } \\
\hline \multicolumn{16}{|l|}{ Chlorophyceae } \\
\hline Ankistrodesmus densus Korshikov* & $\mathrm{S}$ & & & & & & & & & $\mathrm{x}$ & $\mathrm{x}$ & $\mathrm{x}$ & & & $\mathrm{x}$ \\
\hline Ankistrodesmus fusiformis Corda* & S & & & & & & & & & & $\mathrm{x}$ & & & & \\
\hline Asterococcus limneticus Smith ${ }^{*}$ & $\mathrm{~S}$ & & & & & & & & & & & & & $\mathrm{x}$ & \\
\hline Botryosphaera sp.* & $S$ & & & & & & & & & & & $\mathrm{x}$ & $\mathrm{x}$ & & \\
\hline Coelastrum cambricum Archer* & RU & & & & & & $\mathrm{x}$ & & $\mathrm{x}$ & $\mathrm{x}$ & & & $\mathrm{x}$ & $\mathrm{x}$ & $\mathrm{x}$ \\
\hline Coelastrum reticulatum (Dangeard) Senn* & S & $\mathrm{x}$ & & & & & & & & $\mathrm{x}$ & $\mathrm{x}$ & & & & \\
\hline Desmodesmus communis (Hegewald) Hegewald* & $\mathrm{S}$ & & & & & & & & & & $\mathrm{x}$ & & & $\mathrm{x}$ & $\mathrm{x}$ \\
\hline Desmodesmus dispar (Brébisson) Hegewald* & $\mathrm{S}$ & & & & & & & & & & $\mathrm{x}$ & & & & \\
\hline Dictyosphaerium pulchellum Wood* & RU & & $\mathrm{x}$ & & $\mathrm{x}$ & $\mathrm{x}$ & & $\mathrm{x}$ & & $\mathrm{x}$ & $\mathrm{x}$ & $\mathrm{x}$ & $\mathrm{x}$ & $\mathrm{x}$ & $\mathrm{x}$ \\
\hline Elakatothrix linearis Pascher* & S & & & & & & & & & & & & & & $\mathrm{x}$ \\
\hline Eudorina elegans Ehrenberg* & S & & & & & & & & & $\mathrm{x}$ & & $\mathrm{x}$ & $\mathrm{x}$ & & \\
\hline Eudorina sp. ${ }^{*}$ & RU & & $\mathrm{x}$ & & $\mathrm{x}$ & $\mathrm{x}$ & & $\mathrm{x}$ & $\mathrm{x}$ & $\mathrm{x}$ & $\mathrm{x}$ & $\mathrm{x}$ & $\mathrm{x}$ & $\mathrm{x}$ & $\mathrm{x}$ \\
\hline Gloeocystis sp.* & S & & & & & & & & & & & $\mathrm{x}$ & & & \\
\hline Golenkinia radiata Chodat ${ }^{*}$ & RU & & & & & $\mathrm{x}$ & $\mathrm{x}$ & $\mathrm{x}$ & $\mathrm{x}$ & & $\mathrm{x}$ & $\mathrm{x}$ & $\mathrm{x}$ & $\mathrm{x}$ & $\mathrm{x}$ \\
\hline Hydrodictyon reticulatum (Linnaeus) Bory de Saint-Vincent ${ }^{*}$ & RC & $\mathrm{x}$ & $\mathrm{x}$ & $\mathrm{x}$ & $\mathrm{x}$ & $\mathrm{x}$ & $\mathrm{x}$ & $\mathrm{x}$ & $\mathrm{x}$ & $\mathrm{x}$ & $\mathrm{x}$ & $\mathrm{x}$ & $\mathrm{x}$ & & \\
\hline Kirchneriella sp.* & RU & $\mathrm{x}$ & & & & & & & & & $\mathrm{x}$ & $\mathrm{x}$ & $\mathrm{x}$ & $\mathrm{x}$ & $\mathrm{x}$ \\
\hline
\end{tabular}

Continues 
Table 2. Continuation.

\begin{tabular}{|c|c|c|c|c|c|c|c|c|c|c|c|c|c|c|c|}
\hline \multirow{2}{*}{$\begin{array}{l}\text { DIVISION } \\
\text { Class } \\
\text { Genus and species }\end{array}$} & \multirow{2}{*}{$\mathrm{FC}$} & \multicolumn{14}{|c|}{ Sampling sites } \\
\hline & & 厌 & $\bar{z}$ & $\tilde{n}$ & $\tilde{n}$ & $\stackrel{a}{a}$ & $\stackrel{0}{2}$ & $\hat{a}$ & $\stackrel{\infty}{\infty}$ & 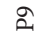 & $\ddot{\simeq}$ & $\widetilde{\simeq}$ & $\approx$ & $\stackrel{\sharp}{\sim}$ & $\stackrel{\mathscr{L}}{\sim}$ \\
\hline Micractinium sp. ${ }^{*}$ & $\mathrm{~S}$ & & & & & & & & & & $\mathrm{x}$ & & & & \\
\hline Microspora sp.* & RU & $\mathrm{x}$ & $\mathrm{x}$ & & & & $\mathrm{x}$ & & $\mathrm{x}$ & $\mathrm{x}$ & & $\mathrm{x}$ & & & \\
\hline Nephrocytium sp.* & S & & & & & & & & & & & & & $\mathrm{x}$ & $\mathrm{x}$ \\
\hline Oedogonium sp.* & $\mathrm{RU}$ & $\mathrm{x}$ & $\mathrm{x}$ & $\mathrm{x}$ & & $\mathrm{x}$ & & & & & & $\mathrm{x}$ & & & \\
\hline Oocystis sp.* & $S$ & & & & & & & & & & & & $\mathrm{x}$ & $\mathrm{x}$ & \\
\hline Pandorina sp. ${ }^{*}$ & S & & & & & & & & & & $\mathrm{x}$ & & $\mathrm{x}$ & & $\mathrm{x}$ \\
\hline Pediastrum duplex Meyen* & RU & & & & & & & & & & & $\mathrm{x}$ & $\mathrm{x}$ & $\mathrm{x}$ & $\mathrm{x}$ \\
\hline Planktosphaeria gelatinosa Smith* & S & & & & & & & & & & & & & & $\mathrm{x}$ \\
\hline Radiococcus sp.* & S & & & & & & & & & & $\mathrm{x}$ & & & & \\
\hline Scenedesmus acuminatus (Lagerheim) Chodat ${ }^{*}$ & $\mathrm{~S}$ & & & & & & & & & & $\mathrm{x}$ & $\mathrm{x}$ & $\mathrm{x}$ & $\mathrm{x}$ & $\mathrm{x}$ \\
\hline Scenedesmus sp.* & S & & & & & & & $\mathrm{x}$ & & & & & & & \\
\hline Sphaerocystis schroeteri Chodat ${ }^{*}$ & S & & & & & & & & & & & $\mathrm{x}$ & $\mathrm{x}$ & & $\mathrm{x}$ \\
\hline Volvox sp. ${ }^{*}$ & $S$ & & & & & & & & & $\mathrm{x}$ & & & & & \\
\hline \multicolumn{16}{|l|}{ Cladophorophyceae } \\
\hline Cladophora sp.* & S & & & $\mathrm{x}$ & & & & & & & & & & & \\
\hline Rhizoclonium sp.* & S & & & & $\mathrm{x}$ & & & & & & $\mathrm{x}$ & $\mathrm{x}$ & & & \\
\hline \multicolumn{16}{|l|}{ Ulvophyceae } \\
\hline Binuclearia sp.* & $\mathrm{S}$ & & & & & & & & & $\mathrm{x}$ & & & & $\mathrm{x}$ & $\mathrm{x}$ \\
\hline \multicolumn{16}{|l|}{ Zygnematophyceae } \\
\hline Actinotaenium wollei (West \& West) Teiling* & RU & & $\mathrm{x}$ & & $\mathrm{x}$ & $\mathrm{x}$ & $\mathrm{x}$ & & & $\mathrm{x}$ & $\mathrm{x}$ & $\mathrm{x}$ & $\mathrm{x}$ & $\mathrm{x}$ & $\mathrm{x}$ \\
\hline Bambusina sp. ${ }^{*}$ & RU & & & & & & $\mathrm{x}$ & & $\mathrm{x}$ & & $\mathrm{x}$ & $\mathrm{x}$ & $\mathrm{x}$ & $\mathrm{x}$ & $\mathrm{x}$ \\
\hline Closterium dianae Ehrenberg ex Ralfs* & S & & & & $\mathrm{x}$ & & $\mathrm{x}$ & & & & & & $\mathrm{x}$ & & \\
\hline Closterium ehrenbergii Meneghini ex Ralfs* & RU & & $\mathrm{x}$ & & $\mathrm{x}$ & $\mathrm{x}$ & & & $\mathrm{x}$ & & $\mathrm{x}$ & $\mathrm{x}$ & $\mathrm{x}$ & & $\mathrm{x}$ \\
\hline Closterium incurvum Brébisson* & S & $\mathrm{x}$ & $\mathrm{x}$ & & $\mathrm{x}$ & & & & & & $\mathrm{x}$ & & $\mathrm{x}$ & & \\
\hline Closterium kuetzingii Brébisson* & RU & & $\mathrm{x}$ & & & $\mathrm{x}$ & & $\mathrm{x}$ & & & $\mathrm{x}$ & $\mathrm{x}$ & $\mathrm{x}$ & $\mathrm{x}$ & $\mathrm{x}$ \\
\hline Closterium lanceolatum Kützing ex Ralfs* & $\mathrm{S}$ & & & & & & & & & & & $\mathrm{x}$ & & & \\
\hline Closterium lineatum Ehrenberg ex Ralfs* & $\mathrm{S}$ & & & & & & & & & & & & & $\mathrm{x}$ & \\
\hline Closterium moniliferum (Bory) Ehrenberg ex Ralfs* & RU & $\mathrm{x}$ & $\mathrm{x}$ & $\mathrm{x}$ & & & $\mathrm{x}$ & $\mathrm{x}$ & $\mathrm{x}$ & & $\mathrm{x}$ & $\mathrm{x}$ & $\mathrm{x}$ & $\mathrm{x}$ & $\mathrm{x}$ \\
\hline Closterium porrectum Nordstedt $^{*}$ & S & & & & & & & & & & & & $\mathrm{x}$ & & \\
\hline Closterium ralfsii Brébisson ex Ralfs* & $\mathrm{S}$ & & & & & & & $\mathrm{x}$ & & & & & $\mathrm{x}$ & & \\
\hline Closterium rostratum Ehrenberg ex Ralfs ${ }^{*}$ & RU & & $\mathrm{x}$ & & $\mathrm{x}$ & $\mathrm{x}$ & $\mathrm{x}$ & $\mathrm{x}$ & $\mathrm{x}$ & $\mathrm{x}$ & $\mathrm{x}$ & & & & $\mathrm{x}$ \\
\hline Closterium setaceum Ehrenberg ex Ralfs* & RU & & & & & & & & & & $\mathrm{x}$ & $\mathrm{x}$ & $\mathrm{x}$ & $\mathrm{x}$ & $\mathrm{x}$ \\
\hline Closterium striolatum Ehrenberg ex Ralfs* & $\mathrm{S}$ & & & $\mathrm{x}$ & $\mathrm{x}$ & & & $\mathrm{x}$ & & & & & & & \\
\hline Closterium tortum Griffiths* & $\mathrm{S}$ & & $\mathrm{x}$ & & & $\mathrm{x}$ & & & & & & & & & \\
\hline Closterium tumidum Johnson* & $\mathrm{S}$ & & & & & & & $\mathrm{x}$ & & & & & & & \\
\hline Cosmarium comissurale Brébisson ex Ralfs* & $\mathrm{S}$ & & & & & $\mathrm{x}$ & & & & & & & $\mathrm{x}$ & & \\
\hline Cosmarium contractum Kirchner* & $\mathrm{S}$ & & & & & & & & & & & $\mathrm{x}$ & & $\mathrm{x}$ & $\mathrm{x}$ \\
\hline Cosmarium lagoense (Nordstedt) Nordstedt* & $\mathrm{S}$ & & & & & & & & & & & & & $\mathrm{x}$ & $\mathrm{x}$ \\
\hline Cosmarium lundelli Delponte ${ }^{*}$ & S & & & & & & & & & & & & & $\mathrm{x}$ & \\
\hline Cosmarium margaritatum (Lundell) Roy \& Bisset ${ }^{\star}$ & $\mathrm{S}$ & & & & & & & & & & & $\mathrm{x}$ & & & \\
\hline
\end{tabular}


Table 2. Continuation.

\begin{tabular}{|c|c|c|c|c|c|c|c|c|c|c|c|c|c|c|c|}
\hline \multirow{2}{*}{$\begin{array}{l}\text { DIVISION } \\
\text { Class } \\
\text { Genus and species }\end{array}$} & \multirow{2}{*}{$\mathrm{FC}$} & \multicolumn{14}{|c|}{ Sampling sites } \\
\hline & & $\vec{I}$ & $\vec{a}$ & $\tilde{\varepsilon}$ & $\tilde{\varepsilon}$ & $\vec{a}$ & $\stackrel{2}{2}$ & $\hat{a}$ & $\stackrel{\infty}{\infty}$ & ฉे & $\vec{\approx}$ & $\widetilde{\simeq}$ & $\mathscr{\sim}$ & $\overleftrightarrow{\simeq}$ & $\stackrel{2}{\sim}$ \\
\hline Cosmarium pachydermum Lundell $^{\star}$ & $S$ & & & & & & & & & & & & & $\mathrm{x}$ & \\
\hline Cosmarium subspeciosum Nordstedt $^{*}$ & S & & $\mathrm{x}$ & $\mathrm{x}$ & & & $\mathrm{x}$ & & & & & & & $\mathrm{x}$ & $\mathrm{x}$ \\
\hline Cylindrocystis sp.* & S & & & & & & & $\mathrm{x}$ & & $\mathrm{x}$ & $\mathrm{x}$ & & & & \\
\hline Desmidium aptogonum Brébisson ex Kützing* & S & & & & & & $\mathrm{x}$ & & & $\mathrm{x}$ & & & & $\mathrm{x}$ & \\
\hline Desmidium baileyi (Ralfs) Nordstedt* & S & & & & & & & & & & & & & $\mathrm{x}$ & $\mathrm{x}$ \\
\hline Desmidium graciliceps (Nordstedt) Lagerheim* & S & & & & & & & & & & & & $\mathrm{x}$ & & \\
\hline Desmidium grevillii (Ralfs) De Bary* & RU & & & & & & & & & & & $\mathrm{x}$ & $\mathrm{x}$ & $\mathrm{x}$ & $\mathrm{x}$ \\
\hline Desmidium laticeps Nordstedt & S & & & & & & & & & & & & & $\mathrm{x}$ & \\
\hline Desmidium quadratum Nordstedt* & RU & & & & & & $\mathrm{x}$ & $\mathrm{x}$ & $\mathrm{x}$ & & $\mathrm{x}$ & $\mathrm{x}$ & $\mathrm{x}$ & $\mathrm{x}$ & $\mathrm{x}$ \\
\hline Desmidium swartzii (Agardh) Agardh ex Ralfs* & S & & & & & & & & & & & & & $\mathrm{x}$ & \\
\hline Euastrum evolutum (Nordstedt) West \& West* & S & & & & & & & & & & $\mathrm{x}$ & & $\mathrm{x}$ & & \\
\hline Euastrum sinuosum Lenormand ex Archer* & S & & & & & & & & & & & & $\mathrm{x}$ & & \\
\hline Euastrum spinulosum Delponte* & S & & & & & & & & & & & & & $\mathrm{x}$ & $\mathrm{x}$ \\
\hline Euastrum subhypochondrum Fritsch \& Rich* & S & & & & & & & & & & & & & & $\mathrm{x}$ \\
\hline Gonatozygon pilosum Wolle* & $\mathrm{RU}$ & & & & & & & & $\mathrm{x}$ & $\mathrm{x}$ & $\mathrm{x}$ & $\mathrm{x}$ & & $\mathrm{x}$ & $\mathrm{x}$ \\
\hline Gonatozygon sp.* & RU & $\mathrm{x}$ & & $\mathrm{x}$ & & $\mathrm{x}$ & & $\mathrm{x}$ & $\mathrm{x}$ & $\mathrm{x}$ & $\mathrm{x}$ & $\mathrm{x}$ & $\mathrm{x}$ & $\mathrm{x}$ & $\mathrm{x}$ \\
\hline Haplozyga sp.* & $S$ & & & & & & & & & & & & & $\mathrm{x}$ & $\mathrm{x}$ \\
\hline Hyalotheca dissiliens Brébisson ex Ralfs* & S & & & & & & & & $\mathrm{x}$ & & & & $\mathrm{x}$ & $\mathrm{x}$ & \\
\hline Hyalotheca mucosa (Mertens) Ehrenberg ex Ralfs* & RU & & & & $\mathrm{x}$ & & $\mathrm{x}$ & $\mathrm{x}$ & $\mathrm{x}$ & $\mathrm{x}$ & $\mathrm{x}$ & $\mathrm{x}$ & $\mathrm{x}$ & $\mathrm{x}$ & $\mathrm{x}$ \\
\hline Mesotaenium sp.* & S & & & & & & & & & & & $\mathrm{x}$ & & & \\
\hline Micrasterias alata Wallich* & S & & & & & & & & & & & & & $\mathrm{x}$ & $\mathrm{x}$ \\
\hline Micrasterias borgei Krieger* & S & & & & & & & & & & & & & $\mathrm{x}$ & \\
\hline Micrasterias furcata Agardh ex Ralfs* & S & & & & & & & & & & & & & $\mathrm{x}$ & $\mathrm{x}$ \\
\hline Micrasterias laticeps Nordstedt $^{*}$ & RU & & $\mathrm{x}$ & $\mathrm{x}$ & $\mathrm{x}$ & $\mathrm{x}$ & & $\mathrm{x}$ & $\mathrm{x}$ & $\mathrm{x}$ & $\mathrm{x}$ & $\mathrm{x}$ & $\mathrm{x}$ & $\mathrm{x}$ & $\mathrm{x}$ \\
\hline Micrasterias pinnatifida (Kützing) ex Ralfs ${ }^{*}$ & $S$ & & & & & & & & & & & & & & $\mathrm{x}$ \\
\hline Micrasterias radians Turner* & $S$ & & & & & & & & & $\mathrm{x}$ & & & & $\mathrm{x}$ & \\
\hline Micrasterias radiosa Ralfs $^{*}$ & $S$ & & & & & & $\mathrm{x}$ & & & & & & & & \\
\hline Micrasterias rotata (Greville) Ralfs* & S & & & & & & $\mathrm{x}$ & & & $\mathrm{x}$ & & $\mathrm{x}$ & $\mathrm{x}$ & & \\
\hline Micrasterias thomasiana Archer* & S & & & & & & & & & $\mathrm{x}$ & & $\mathrm{x}$ & & & $\mathrm{x}$ \\
\hline Micrasterias torreyi Bailey ex Ralfs* & S & & & & & & & & & & & & $\mathrm{x}$ & $\mathrm{x}$ & \\
\hline Micrasterias truncata (Corda) Brébisson ex Ralfs ${ }^{*}$ & S & & & & & & & & & & & $\mathrm{x}$ & & & \\
\hline Mougeotia sp.* & $\mathrm{RC}$ & $\mathrm{x}$ & $\mathrm{x}$ & & & $\mathrm{x}$ & $\mathrm{x}$ & $\mathrm{x}$ & $\mathrm{x}$ & $\mathrm{x}$ & $\mathrm{x}$ & $\mathrm{x}$ & $\mathrm{x}$ & $\mathrm{x}$ & $\mathrm{x}$ \\
\hline Onychonema laeve Nordstedt* & S & & & & & & & & & & & $\mathrm{x}$ & & $\mathrm{x}$ & $\mathrm{x}$ \\
\hline Pleurotaenium coronatum (Brébisson ex Ralfs) ${ }^{*}$ & S & & & & & & & & & & $\mathrm{x}$ & & & & $\mathrm{x}$ \\
\hline Pleurotaenium ehrenbergii (Brébisson ex Ralfs) Delponte* & S & & & & & & & $\mathrm{x}$ & $\mathrm{x}$ & & & & & & \\
\hline Pleurotaenium subcoronulatum (Turner) West $\&$ West ${ }^{*}$ & S & & & & & & & & & & & $\mathrm{x}$ & & & \\
\hline Pleurotaenium subundulatum Borge* & S & & & & & & & & & & & & & $\mathrm{x}$ & \\
\hline Pleurotaenium trabecula (Ehrenberg) ex Nägeli* & S & & & & & & & & $\mathrm{x}$ & & & & & $\mathrm{x}$ & \\
\hline Sirogonium sp.* & S & & & & & & & & $\mathrm{x}$ & & & & & & \\
\hline Spirogyra sp.* & $\mathrm{RU}$ & $\mathrm{x}$ & $\mathrm{x}$ & $\mathrm{x}$ & $\mathrm{x}$ & & & & $\mathrm{x}$ & & $\mathrm{x}$ & $\mathrm{x}$ & $\mathrm{x}$ & $\mathrm{x}$ & $\mathrm{x}$ \\
\hline
\end{tabular}

Continues 
Table 2. Continuation.

\begin{tabular}{|c|c|c|c|c|c|c|c|c|c|c|c|c|c|c|}
\hline \multirow{2}{*}{$\begin{array}{l}\text { DIVISION } \\
\text { Class } \\
\text { Genus and species }\end{array}$} & \multirow{2}{*}{ FC } & \multicolumn{13}{|c|}{ Sampling sites } \\
\hline & & $\vec{I}$ & $\vec{a}$ & $\stackrel{n}{a}$ & $\tilde{n}$ & $\ddot{2}$ & $\stackrel{\circ}{2}$ & $\hat{\imath} \stackrel{\infty}{\curvearrowright}$ & 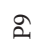 & $\ddot{\simeq}$ & $\approx$ & $\approx$ & $\ddot{\simeq}$ & $\stackrel{2}{\sim}$ \\
\hline Spirotaenia sp.* & S & & & & & & & $\mathrm{x}$ & & & & & & \\
\hline Spondylosium moniliforme Lundell ${ }^{*}$ & S & & & & & & & & & & $\mathrm{x}$ & & $\mathrm{x}$ & \\
\hline Spondylosium panduriforme (Heimerl) Teiling* & S & & & & & & & & & & & & $\mathrm{x}$ & \\
\hline Spondylosium planum (Wolle) West \& West ${ }^{*}$ & S & & & & & & & & & & $\mathrm{x}$ & $\mathrm{x}$ & & $\mathrm{x}$ \\
\hline Spondylosium pulchrum (Bailey) Archer* & S & & & & & & & & & & & & $\mathrm{x}$ & $\mathrm{x}$ \\
\hline Staurastrum asteroideum West \& West ${ }^{*}$ & S & & & & $\mathrm{x}$ & $\mathrm{x}$ & & & & & & & $\mathrm{x}$ & \\
\hline Staurastrum boergesenii Raciborski & S & & & & & & & & & & & & $\mathrm{x}$ & \\
\hline Staurastrum coarctatum Brébisson* & S & & & & & & & & & & $\mathrm{x}$ & & $\mathrm{x}$ & $\mathrm{x}$ \\
\hline Staurastrum columbetoides West $\&$ West ${ }^{*}$ & S & & & & & & & & & & & & $\mathrm{x}$ & \\
\hline Staurastrum grallatorium Nordstedt & RU & & & & & & & & & & $\mathrm{x}$ & & $\mathrm{x}$ & $\mathrm{x}$ \\
\hline Staurastrum hagmannii Grönblad ${ }^{*}$ & S & & & & & & & & & & & & $\mathrm{x}$ & $\mathrm{x}$ \\
\hline Staurastrum leptacanthum Nordstedt & RU & & & & & & & & & & $\mathrm{x}$ & $\mathrm{x}$ & $\mathrm{x}$ & $\mathrm{x}$ \\
\hline Staurastrum leptocladum Nordstedt $^{*}$ & RU & & & & & & & & & & $\mathrm{x}$ & $\mathrm{x}$ & $\mathrm{x}$ & $\mathrm{x}$ \\
\hline Staurastrum manfeldtii Delponte ${ }^{*}$ & RU & & & & & & & & & & & & $\mathrm{x}$ & $\mathrm{x}$ \\
\hline Staurastrum margaritaceum (Ehrenberg) Ralfs* & $\mathrm{RU}$ & & $\mathrm{x}$ & & $\mathrm{x}$ & $\mathrm{x}$ & & & & & $\mathrm{x}$ & & $\mathrm{x}$ & $\mathrm{x}$ \\
\hline Staurastrum minnesotense Wolle ${ }^{*}$ & S & & & & & & & & & & & $\mathrm{x}$ & & \\
\hline Staurastrum novae-caesareae Wolle & S & & & & & & & & & & & & $\mathrm{x}$ & $\mathrm{x}$ \\
\hline Staurastrum ophiura Lundell ${ }^{*}$ & S & & & & & & & & & & & & $\mathrm{x}$ & \\
\hline Staurastrum oxyacanthum Archer* & S & & & & & & & & & & & & $\mathrm{x}$ & \\
\hline Staurastrum penicilliferum Grönblad ${ }^{*}$ & S & & & & & & & & & & & & $\mathrm{x}$ & \\
\hline Staurastrum quadrangulare (Brébisson) Ralfs & S & & & & & & & & & & $\mathrm{x}$ & & & \\
\hline Staurastrum rotula Nordstedt & $\mathrm{RU}$ & & & & & & & & & & $\mathrm{x}$ & & $\mathrm{x}$ & $\mathrm{x}$ \\
\hline Staurastrum setigerum Cleve & RU & $\mathrm{x}$ & $\mathrm{x}$ & $\mathrm{x}$ & $\mathrm{x}$ & $\mathrm{x}$ & $\mathrm{x}$ & $\mathrm{x}$ & $\mathrm{x}$ & $\mathrm{x}$ & $\mathrm{x}$ & $\mathrm{x}$ & $\mathrm{x}$ & $\mathrm{x}$ \\
\hline Staurastrum subindentatum West \& West* & S & & & & & & & & & & & & $\mathrm{x}$ & \\
\hline Staurastrum trifidum Nordstedt & S & & & & & & & & & & $\mathrm{x}$ & & $\mathrm{x}$ & $\mathrm{x}$ \\
\hline Staurastrum vestitum Ralfs $^{\star}$ & S & & & & & & & & & & & $\mathrm{x}$ & $\mathrm{x}$ & $\mathrm{x}$ \\
\hline Staurastrum wolleanum Butler* & S & & & & & & & & & & $\mathrm{x}$ & & $\mathrm{x}$ & $\mathrm{x}$ \\
\hline Staurodesmus bulnheimii (Raciborski) Round \& Brook ${ }^{\star}$ & S & & & & & & & & & & & & & $\mathrm{x}$ \\
\hline Staurodesmus connatus (Lundell) Thomasson ${ }^{*}$ & S & & & & & & & & & & $\mathrm{x}$ & & $\mathrm{x}$ & \\
\hline Staurodesmus convergens (Ehrenberg ex Ralfs) Teiling & S & & & & & & & & & & $\mathrm{x}$ & $\mathrm{x}$ & & $\mathrm{x}$ \\
\hline Staurodesmus cuspidatus (Brébisson) Teiling & S & & & & & & & & & & & & $\mathrm{x}$ & \\
\hline Staurodesmus leptodermus (Lundell) Thomasson* & S & & & & & & & & & $\mathrm{x}$ & & & & \\
\hline Staurodesmus lobatus (Börgesen) Bourrelly* & S & & & & & & & & & & & & $\mathrm{x}$ & $\mathrm{x}$ \\
\hline Staurodesmus mamillatus (Nordstedt) Teiling* & RU & & & & & & & & & & $\mathrm{x}$ & $\mathrm{x}$ & $\mathrm{x}$ & $\mathrm{x}$ \\
\hline Staurodesmus selenaeus (Grönblad) Teiling* & S & & & & & & & & & & & & $\mathrm{x}$ & \\
\hline Triploceras gracile Bailey* & S & & & & & & & & & & & & $\mathrm{x}$ & $\mathrm{x}$ \\
\hline Xanthidium antilopaeum (Brébisson) Kützing* & RU & & & & & & & & & & & $\mathrm{x}$ & $\mathrm{x}$ & $\mathrm{x}$ \\
\hline Xanthidium regulare Nordstedt ${ }^{*}$ & S & & & & & & & & & & & $\mathrm{x}$ & $\mathrm{x}$ & \\
\hline Zygnema sp.* & $\mathrm{RU}$ & $\mathrm{x}$ & & $\mathrm{x}$ & & $\mathrm{x}$ & & $\mathrm{x}$ & & $\mathrm{x}$ & $\mathrm{x}$ & $\mathrm{x}$ & & $\mathrm{x}$ \\
\hline
\end{tabular}

FC - frequency class; S - sporadic; RU - relatively uncommon; RC - relatively common; QC - quite common.

${ }^{\star}$ New records; ${ }^{* *}$ Heterokontophyta, according to Hoek et al. (1995). 


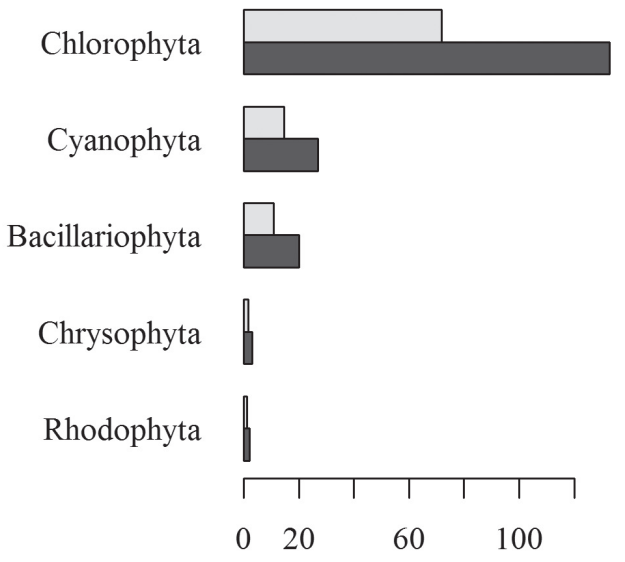

Proportion and number of taxa

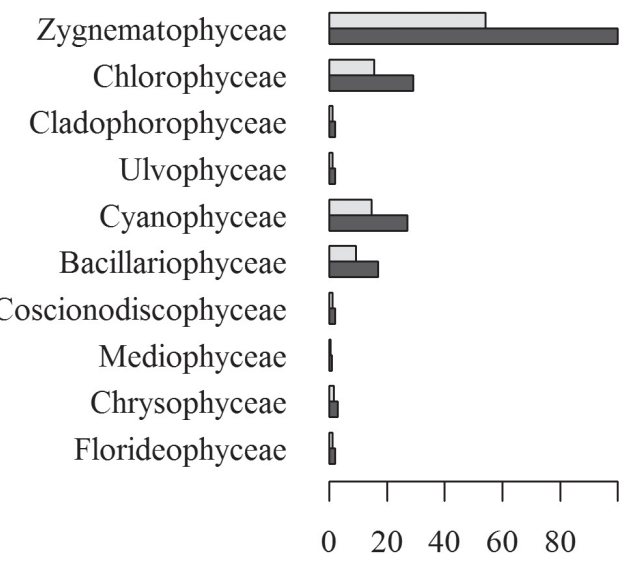

Proportion and number of taxa

Figure 2. Distribution of taxa by division (left side) and class (right side), showing frequencies (light bars) and absolute values (grey bars).

As shown in Fig. 2, the division Chlorophyta was represented by the classes Zygnematophyceae (54.05\%), Chlorophyceae (15.68\%), Cladophorophyceae (1.08\%) and Ulvophyceae (1.08\%); Cyanophyta was represented by the class Cyanophyceae (14.59\%); Bacillariophyta was represented by the classes Bacillariophyceae $(9.19 \%)$, Coscinodiscophyceae (1.08\%) and Mediophyceae (0.54\%); Chrysophyta was represented by the class Chrysophyceae (1.62\%); and Rhodophyta was represented by the class Florideophyceae (1.08\%).

The class Zygnematophyceae had the most taxa $(\mathrm{n}=$ $100)$, followed by Chlorophyceae $(n=29)$. Those two classes collectively accounted for $70 \%$ of the taxa, which explains the high representation of the division Chlorophyta. The group Cladophorophyceae had the smallest number of representatives $(\mathrm{n}=2)$.

The most common taxa (those categorized as quite common or relatively common) in the Araguari and Falsino rivers were Surirella linearis (89.0\%), Leptolyngbya sp. (84.0\%), Rhipidodendron huxleyi (71.0\%), Fragilaria sp. (68.0\%), Eunotia yanomami (61.0\%), Batrachospermum sp. (59.0\%), Hydrodictyon reticulatum (55.0\%), Surirella grunowii (54.0\%), Mougeotia sp. (45.0\%), and Tabellaria sp. (45.0\%). Five of the ten most common species belonged the group Bacillariophyta.

The majority of taxa showed a low frequency: $69.0 \%$ were categorized as sporadic and $25.0 \%$ were categorized as relatively uncommon. Only $4.0 \%$ and $2.0 \%$ of the taxa were categorized as relatively common and quite common, respectively (Fig. 3).

Fig. 4 shows the spatial distribution of taxa. The sites with the highest richness were R4 ( $\mathrm{n}=96), \mathrm{R} 5(\mathrm{n}=92), \mathrm{R} 2$ $(\mathrm{n}=77), \mathrm{R} 3(\mathrm{n}=69)$, and R1 $(\mathrm{n}=57)$. Those same sites (R2-R5) were grouped together in the cluster analysis (Fig. 5). The site with the smallest number of taxa was $\mathrm{P} 4$, with only 33 taxa. As can be seen in Fig. 6, species richness was highest $(\mathrm{n}=132)$ during the dry season (November) and lowest $(n=73)$ during the dry-wet seasonal transition (February).

As previously mentioned, there have been only two studies on continental microalgae conducted in the state of Amapá (Förster 1964; Souza \& Melo 2011). Our study had 11 taxa in common with those two works (Tab. 3). Therefore, of the 185 taxa identified in the present study, 174 represent new records for the state (Tab. 2).

\section{Discussion}

The division Chlorophyta had the highest number of taxa, which occurred mostly in the CNHP reservoir. This environment provided conditions that were more favorable for the development of the phytoplankton than were those elsewhere on the river, which is quite turbulent, a condition to which few species can adapt. Other rivers with dams display this same pattern: the Contas river, in the state of Bahia (Fuentes et al. 2010); the Corumbá river, in the state of Goiás (Silva et al. 2001); and the Paranapanema river, in the southeast of Brazil (Nogueira et al. 2010).

The class Zygnematophyceae had the largest number of taxa. That is probably attributable the characteristics of the two studied rivers. According to Coesel (1982), oligotrophic waters, with the presence of macrophytes and low electric conductivity, have a high richness of desmids. Bárbara et al. (2010) and Cunha et al. (2011) both classified the Araguari and Falsino rivers as oligotrophic and found that the CNHP reservoir, where desmids were more numerous, had aquatic macrophytes. In the Amazon basin, there is considerable desmid diversity, making it one of the best known groups in the region (Grönblad 1945; Förster 1963, 1964, 1969, 1974; Scott et al. 1965; Thomasson 1971; 


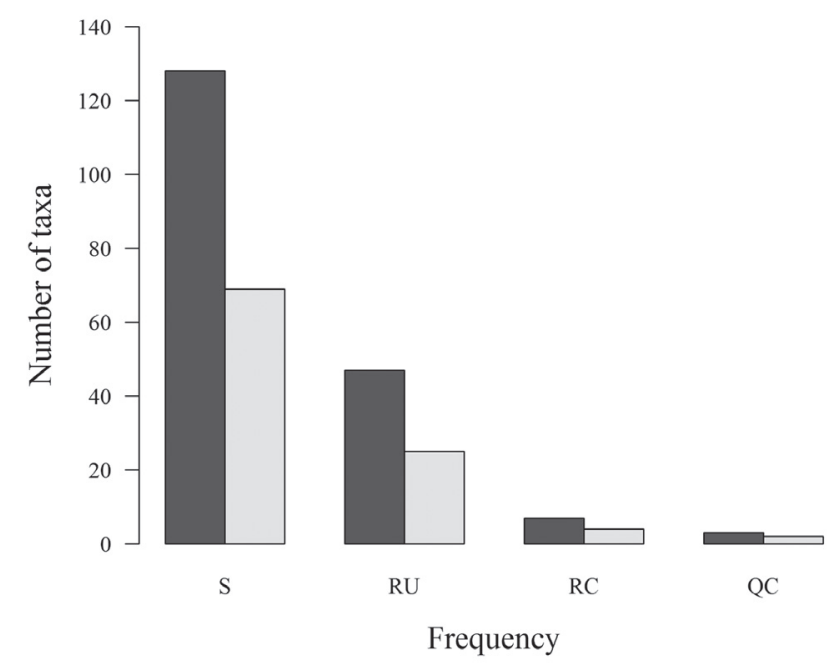

Figure 3. Distribution of taxa by frequency, showing frequencies (light bars) and absolute values (grey bars).

$\mathrm{S}$ - sporadic; RU - relatively uncommon; RC - relatively common; QC - quite common.

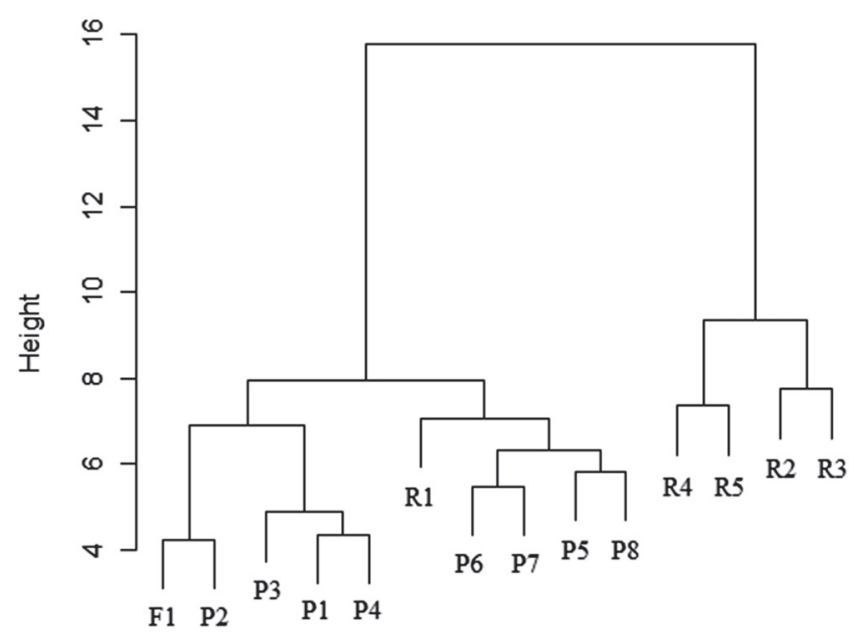

Figure 5. Cluster analysis: groupings according to the similarity of species composition among the sampling sites.

Sophia \& Huszar 1996; Lopes \& Bicudo 2003; Melo \& Souza 2009; Souza \& Melo 2011).

The majority of the most common taxa belonged to the division Bacillariophyta. This result was expected, because diatoms are typical of predominantly lotic environments (Soares et al. 2007). These organisms did not exhibit the

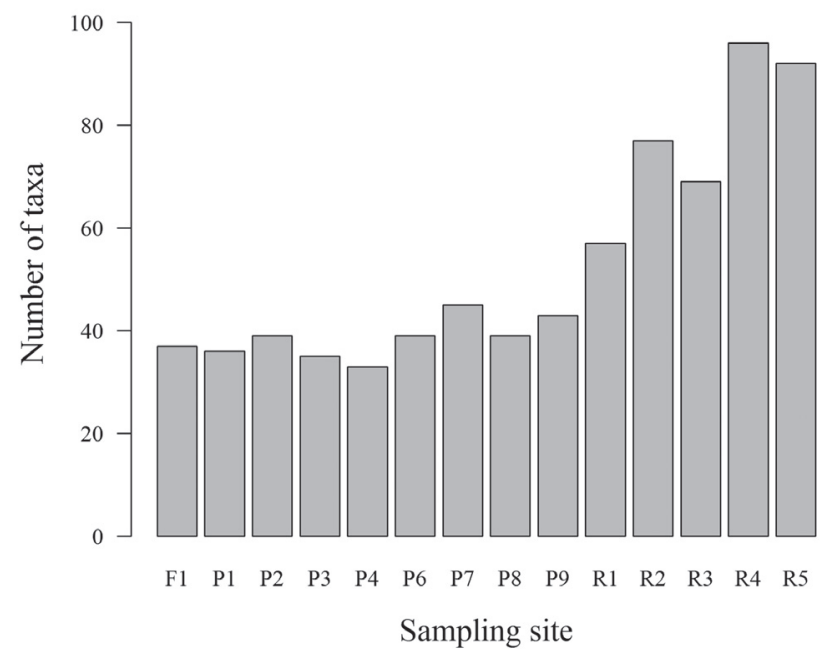

Figure 4. Distribution of taxa among the sampling sites.

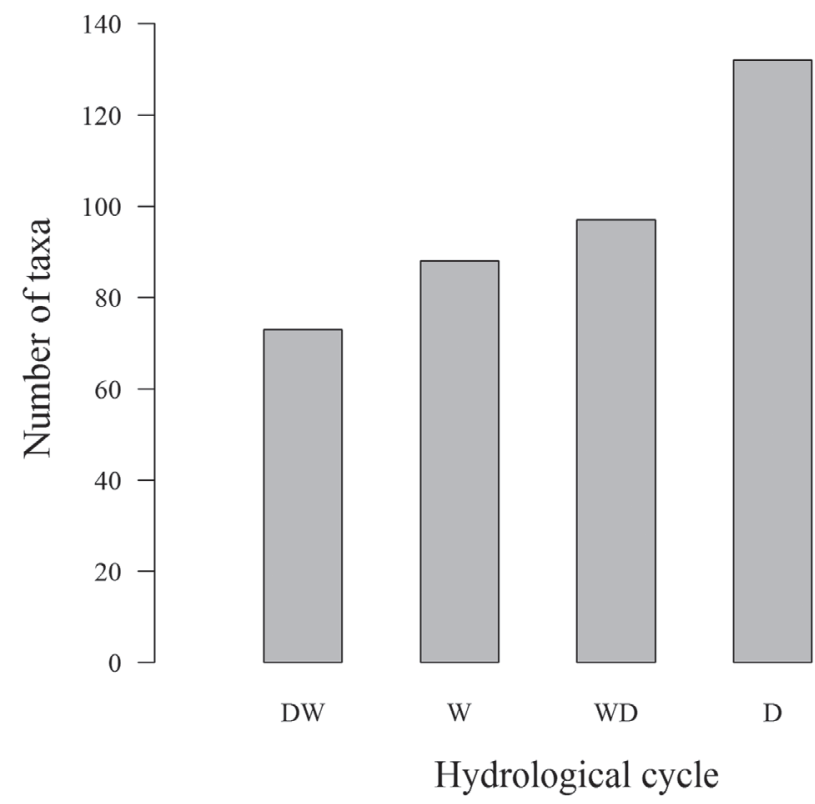

Figure 6. Distribution of taxa by hydrological period.

DW - dry-wet seasonal transition; W - wet season; WD - wet-dry seasonal transition; and D - dry season.

highest richness, but they occurred in almost all samples. In a study conducted on the Arataï river in French Guiana, which shares a border with the state of Amapá, Tudesque et al. (2012) reported that Surirella sp., the most common genus in our study, is adapted to the turbulence of rivers and possesses high mobility. Furthermore, these authors 
Table 3. Previous studies of continental microalgae in the state of Amapá: taxa in common with the present study.

\begin{tabular}{ccc}
\hline Reference & Taxa in common with the present study \\
\hline Sampling site & Number & Species \\
Förster (1963) & 1 & Desmidium laticeps \\
Oiapoque river & 10 & $\begin{array}{c}\text { Staurastrum boergesenii; Staurastrum grallatorium; Staurastrum leptacanthum; } \\
\text { Staurastrum novae-caesareae; Staurastrum quadrangulare; Staurastrum rotula; } \\
\text { Staurastrum setigerum; Staurastrum trifidum; Staurodesmus convergens; } \\
\text { and Staurodesmus cuspidatus }\end{array}$ \\
\hline
\end{tabular}

observed the diatoms Eunotia sp., Fragilaria sp. and Surirella sp. in high frequency, similar to our findings on the Araguari and Falsino rivers. Diatoms have also been reported to be the most common group on other Amazonian rivers such as the Guamá and Caeté, in the state of Pará (Monteiro et al. 2009; Pereira et al. 2010).

The high frequency of diatoms in the present study might be explained by their ability to adapt to conditions of high turbulence (Reynolds et al.1994), a typical characteristic of rivers (Press et al. 2003). For the stretch of river studied Cunha et al. $(2010 ; 2011)$ reported the mean water velocity to be $0.57-0.92 \mathrm{~m} / \mathrm{s}$ in the lotic stretch (upstream) and $0.04 \mathrm{~m} / \mathrm{s}$ in the lentic stretch (CNHP reservoir). Branco et al. (1986) categorized the water velocity as: quite rapid $(>0.5 \mathrm{~m} / \mathrm{s})$, or moderate-to-slow $(\leq 0.5 \mathrm{~m} / \mathrm{s})$. Therefore, the studied stretch of river can be considered mostly turbulent because of its high water velocity, which tends to naturally decrease toward the dam. In the CNHP reservoir, however, the speed is not uniform as in totally lentic environments, because its water retention time is only two to five days, and its characteristics therefore resemble those of a river more than those of a lake (Bárbara et al. 2010; Cunha et al. 2013).

In the present study, most of the taxa were classified as sporadic, occurring in less than $10 \%$ of the samples. According to Townsed et al.(2008), this pattern is normal, because these species in general are naturally rare. In studies conducted on the Guamá and Paranaíba rivers (Monteiro et al. 2009; Oliveira et al. 2011), the majority of taxa were also classified as sporadic.

The spatial distribution of taxa was aggregate in the CNHP reservoir. The majority of these taxa are desmids, which present slow growth and require lentic environments for their development (Coesel, 1982). The dendrogram of the cluster analysis (Fig. 5) indicated one cluster associated with the reservoir sampling sites and two additional large clusters, one associated with the sites near the beginning of the stretch of river studied and another associated with sites intermediate between the two ends of the stretch. Considering the species composition, the locations of these clusters might be related to the declivity of the land at the sampling sites and, consequently, to the water velocity, the declivity being similar among sites within the same group. The sites at the beginning of the stretch (F1 and P1-P4) had high declivity, the remaining sites along the stretch (P6-P9 and R1) had intermediate declivity, and the sites at the reservoir had low declivity, resulting in low water velocity (Cunha et al. 2011), the last creating the most favorable conditions for the development and reproduction of algae (Coesel, 1982).

Because of the presence of a reservoir in the stretch of river studied, we expected more taxa of the division Cyanophyta, or that their distribution would be more restricted to the reservoir, because these organisms typically occur in lentic environments, with a long water retention time (Chorus \& Bartram, 1999). However, the CNHP reservoir has a water retention time of two to five days; that is, all the water in the reservoir is renewed in a relatively short period of time, even when considering the complete hydrological cycle (Cunha et al. 2011). Much longer water retention times have been reported for the reservoirs of other hydroelectric plants in the Amazon basin (Tucci, 1998): 50, 107 and 578 days, respectively, at the Tucuruí, Samuel and Balbina reservoirs. This fast turnover prevents the occurrence of algal blooms, which, according to Chorus \& Bartram (1999) do not usually occur in environments where the water retention time is shorter than five days. However, these characteristics might be altered with the construction of new reservoirs, and two additional hydroelectric plants are currently under construction on the stretch of river studied (Cunha et al. 2010; 2011; 2013). In fact, the water retention time might increase considerably. On rivers with dams, such as the Paraibuna river (in the state of Minas Gerais) and the Paranapaíba river (in the states of Goiás and Minas Gerais), Cyanophyta is the most well-represented division, in terms of the number of species (Soares et al. 2007; Oliveira et al. 2011). The increase in species richness seen during the dry season in the present study might be related to the physical characteristics of that season, such as greater solar radiation, higher temperatures and less precipitation, all of which favor the growth of algae (Kivrak 2006; Marques et al. 2012). In addition, water velocity tends to decrease during the dry season (Nogueira 2000; Zeng et al. 2006; Cunha et al. 2013). 
The occurrence of a larger number of taxa during the dry season has also been reported for other Brazilian rivers, such as the Urubuí (Aprile \& Mera 2007), Paraibuna and Pomba (Soares et al. 2007).

Reviewing the literature, we found only two studies of phytoplankton in the state of Amapá: Förster (1964), who studied desmids on the Oiapoque river and recorded five taxa; and Souza \& Melo (2011), who conducted a survey of desmids in Amapá lake and recorded 35 taxa. Therefore, in the present study, we have identified the largest number of taxa recorded to date for the state of Amapá (185), of which $172(93 \%)$ represent new records for the state.

\section{Conclusion}

We found that the species richness of the phytoplankton community of the Araguari and Falsino rivers was high in comparison with that reported in similar studies for the Amazon and for other regions of Brazil. This suggests that, in addition to the natural tendency toward an increase in species richness toward the tropics, the sampling effort was satisfactory, because we identified spatio-temporal variations within the microalgae community. We observed a large number of taxa belonging to the division Chlorophyta, class Zygnematophyceae, and most of the taxa identified were relatively rare. However, most of the taxa classified as common were diatoms. When we examined the spatiotemporal dynamics, we found that species richness was lowest at the sampling sites close to conservation units (sites with high declivity and high water velocity) and increased progressively at each site nearer to the CNHP reservoir (sites at which the declivity and water velocity were low). In addition, the number of taxa was highest in dry season. The 174 new records generated for the state of Amapá constitute a significant contribution to expanding the knowledge of aquatic biodiversity in the Brazilian Amazon.

\section{Acknowledgments}

This study received financial support from the Brazilian Conselho Nacional de Desenvolvimento Científico e Tecnológico (CNPq, National Council for Scientific and Technological Development; Master's Scholarship Grant to E.D.S.C. and Research Grant no. 305657/2009-7).

\section{References}

Agostinho, A.A.; Thomaz, S.M. \& Gomes, L.C. 2005. Conservação da biodiversidade em águas continentais do Brasil. Megadiversidade 1: 70-78.

Aprile, F.M. \& Mera, P.A.S. 2007. Fitoplâncton e fitoperifíton de um rio de águas pretas da Amazônia Periférica do Norte, Brasil. Brazilian Journal Aquatic Science and Technology 11(2): 1-14.

Almeida, F.F. \& Melo, S. 2011. Phytoplankton community structure in an Amazon floodplain lake (Lago Catalão, Amazonas, Brazil). Neotropical Biology and Conservation 6(2):112-123.
Araújo-Lima, C.A.R.M.; Forsberg, B.R.; Victoria, R. \& Martinelli, L. 1986 Energy sources for detritivorous fishes in the Amazon. Science 234 1256-1258.

Bárbara,V.F.; Cunha, A.C.; Rodrigues, A.S.L. \& Siqueira, E.Q. 2010. Monitoramento Sazonal da qualidade da água do rio Araguari/AP. Revista Biociências 16(1): 57-72.

Bicudo, C.E.M. \& Menezes, M. 2006. Gêneros de Algas de Águas Continentais do Brasil. São Carlos, Rima.

Bicudo, C.E.M. \& Menezes, M. 2010. Algas do Brasil. Pp: 49-60. In: Forzza, R.C.; Leitman, P.M.; Costa, A.; Carvalho-Júnior, A.A.; Peixoto, A.L.; Walter, B.M.T.; Bicudo, C.; Zappi, D.; Costa, D.P.; Lleras, E.; Martinelli, G.; Lima, H.C.; Prado, J.; Stehmann, J.R.; Baumgratz, J.F.A.; Pirani, J.R.; Sylvestre, L.S.; Maia, L.C.; Lohmann, L.G.; Paganucci, L.; Silveira, M.; Nadruz, M.; Mamede, M.C.H.; Bastos, M.N.C.; Morim, M.P.; Barbosa, M.R.; Menezes, M.; Hopkins, M.; Secco, R.; Cavalcanti, T. \& Souza, V.C. (Eds.). Catálogo de Plantas e Fungos do Brasil. Vol 1. Rio de Janeiro.

Bozelli, R.L. \& Huszar, V.L.M. 2003. Comunidades fito e zooplanctônicas continentais em tempo de avaliação. Limnotemas 3. Sociedade Brasileira de Limnologia.

Branco, S. M. 1986. Hidrobiologia Aplicada à Engenharia Sanitária. São Paulo, Cetesb.

Brasil. CONAMA. 2005. Resolução No. 357, de 17 de março de 2005. Dispõe sobre a classificação dos Corpos de Água e Diretrizes Ambientais para o seu Enquadramento, bem como Estabelece as Condições e Padrões de Lançamento de Efluentes.

Brito, D.M.C. 2008. Conflitos em unidades de conservação. Revista Eletrônica de Humanidades do Curso de Ciências Sociais da UNIFAP 1

Chorus, I. \& Bartram, J. 1999. Toxic Cyanobacteria in water: a guide to their public health consequences, monitoring and management E \& FN Spon, London.

Coesel, P.F.M. 1982. Structural characteristics and adaptations of desmid communities. Journal of Ecology 70: 163-177.

Cunha, A.C.; Cunha, H.F.A.; Brasil Júnior, A.C.P.; Daniel, L.A. \& Schulz, H.E. 2004. Qualidade microbiológica em rios de áreas urbanas e periurbanas no Baixo Amazonas: o caso do Amapá. Revista Brasileira de Engenharia Sanitária e Ambiental 9(4): 322-328.

Cunha, A.C.; Cunha, H.F.A.; Souza, J.A.; Nazaré, A.S. \& Pantoja, S. 2005. Monitoramento de águas superficiais em rios estuarinos do Estado do Amapá sob poluição microbiológica. Boletim do Museu Paraense Emílio Goeldi (MPEG), Série Ciências Naturais 1(1): 191-199.

Cunha, A.C.; Brito, D.C. \& Cunha, H.F.A. 2010. Análise de Cenário da Qualidade da Água no Rio Araguari (AP) com Uso do Sistema de Modelagem QUAL2Kw: Impactos de Hidrelétricas e Urbanização. Pp: 135- 154 In: Cunha, A.C.; Souza, E.B. \& Cunha, H.F.A. (Eds.). Tempo, Clima e Recursos Hídricos. Macapá, Editora IEPA.

Cunha, A.C.; Brito, D.C.; Cunha, H.F.A. \& Schulz, H.E. 2011. Dam Effect on Stream Reaeration Evaluated with the Qual2kw Model: Case Study of the Araguari River, Amazon Region, Amapá State/Brazil. Pp: 153-177. In: Bilibio, C.; Hensel, O. \& Selbach, J. (Eds.). Sustainable water management in the tropics and subtropics - and case studies in Brazil. Vol 2. Jaguarão.

Cunha, A.C.; Pinheiro, L.A.R. \& Cunha, H.F.A. 2013. Modelagem e simulação do escoamento e dispersão sazonais de agentes passivos no rio araguari - AP: cenários para o AHE Ferreira Gomes-I- Amapá/Brasil. Revista Brasileira de Recursos Hídricos 18(1): 34-51

Diaz-Castro, J.G; Souza-Mosimann, R.M.; Laudares-Silva, R. \& Forsberg, B. Rider. 2003. Composição da comunidade de diatomáceas periféricas do rio Jaú, Amazonas, Brasil. Acta Amazonica 33(4): 583-606.

Ferrari, F.; Procopiak, L.K.; Alencar, Y.B \& Ludwig, T.A. V. 2007. Eunotiaceae (Bacillariophyceae) em igarapés da Amazônia Central, Manaus e Presidente Figueiredo, Brasil. Acta Amazonica 37(1): 1-16.

Förster, K. 1963. Desmidiaceen aus Brasilien, 1: Nord Brasilien. Revue Algologique 7(1): 38-92.

Förster, K. 1964. Desmidiaceen aus Brasilien, 2: Bahia, Goyaz, Piauhi und Nord Brasilien. Hidrobiologia 23(3-4): 321-505.

Förster, K. 1969. Amasonische Desmidiaceen, 1: Area 1 Santarém. Amazoniana 2(1-2): 5-231 
Förster, K. 1974. Amazonische Desmidiaceen, 2, Area 1 Maués-Abacaxis. Amazoniana 5(2): 135-242.

Fuentes, E.V.; Oliveira, H.S.B.; Araujo, M.K.C.; Severi, W. \& Moura, A.N. 2010. Variação espacial e temporal do fitoplâncton do rio de Contas, Bahia, Brasil. Revista Brasileira de Engenharia de Pesca 5(2): 13-25.

Grönblad, R. 1945. Brazilian algae, specially Desmidiaceae from low region of Amazon river from August Ginzberger samples on year of MCMXXVII. Acta Societatis Scientiarum Fennicae, Nova Séries B, 2: 1-42.

Hoek, C. van-de; Mann, D.G. \& Janhs, H.M. 1995. Algae: an introduction to phycology. Cambridge, University of Cambridge Press.

Huszar, V.L.M. \& Giane, A. 2007. Amostragem da comunidade fitoplânctonica em águas continentais: reconhecimento e padrões espaciais e temporais. Pp: 133-147. In: Bicudo \& Bicudo. Amostragem em limnologia. São Carlos, Editora Rima.

Huszar, V.L.M. \& Reynolds, C.S. 1997. Phytoplankton periodicity and sequences of dominance in an Amazonian flood-plain lake (Lago Batata, Pará, Brasil): responses to gradual environmental change. Hydrobiologia 346: 169-181.

Ibañez, M.S.R. 1998. Phytoplankton composition and abundance of a central Amazonian floodplain lake. Hydrobiologia 362: 79-83.

Junk, W.J.; Piedade, M.T.F.; Schöngart, J. \& Wittmann, F. 2012. A classification of major natural habitats of Amazonian white-water river floodplains (várzeas). Wetlands Ecology and Management 20(6): 461-475.

Kivrak, E. 2006. Seasonal and long term changes of the phytoplankton in the lake Tortum in relation to environmental factors, Erzurum, Turkey. Biologia, Bratislava 61(4): 339-345.

Komárek, J.; Anagnostidis, K. 1999. Cyanoprokaryota, 1: Chroococcales. In: Süsswasserflora Von Mitteleuropa. Jena. Gustav Fischer.

Komárek, J. \& Anagnostidis, K. 2005. Cyanoprokaryota, 2: Oscillatoriales. In: Süsswasserflora von Mitteleuropa. München. Elsevier.

Lopes, M.R.M. \& Bicudo, C.E.M. 2003. Desmidioflórula de um lago da planície de inundação do rio Acre, Estado do Amazonas, Brasil. Acta Amazônica 33(2): 167- 212

Marques, D.D.; Brito, A.U.; Cunha, A.C. \& Souza, L.R. 2012. Variação da radiação solar no estado do Amapá: estudo de caso em Macapá, Pacuí, Serra do Navio e Oiapoque no período de 2006 a 2008. Revista Brasileira de Meteorologia 27(2): 127 - 138.

Mateucci, S.D. \& Colma, A. 1982. La metodologia para el estudo de la vegetácion. Washington, Collection de Monografias Cientificas 22.

Medlin, L.K. \& Kaczmarska, I. 2004. Evolution of the diatoms: V. Morphological and cytological support for the major clades and a taxonomic revision. Phycologia 43(3): 245-270.

Melo, S. \& Huszar, V.L.M. 2000. Phytoplankton in an Amazonian floodplain lake (Lago Batata, Brasil): diel variation and species strategies. Journal of Plankton Research 22(1): 63-76.

Melo, S.; Rebelo, S.R.M.; Souza, K.F.; Menezes, M. \& Torgan, L.C. $2005 a$. Fitoplâncton. Pp: 87-98. In: Santos-Silva, E.N. et al. (Ed.). Biotupé: Meio Físico, Diversidade Biológica e Sociocultural do Baixo Rio Negro, Amazônia Central. Manaus.

Melo, S.; Rebelo, S.R.M.; Souza, K.F.; Soares, C.C. \& Sophia, M.G. 2005 b. Desmídias com ocorrência planctônica. Pp. 99-108. In: Santos-Silva, E.N. et al. (Eds.) Biotupé: Meio Físico, Diversidade Biológica e Sociocultural do Baixo Rio Negro, Amazônia Central. Manaus.

Melo, S.; Souza, K.F.; Rebelo, S.R.M. \& Sophia, M.G. 2009. Gêneros Euastrum Ehrenberg ex Ralfs e Micrasterias C. Agardh (Conjugatophyceae-Desmidiaceae) de dois ambientes amazônicos de águas pretas (Manaus, Amazonas-Brasil). Acta Amazonica 39(1): 13-19.

Melo, S. \& Souza, K.F. 2009. Flutuação anual e interanual da riqueza de espécies de desmídias (Chlorophyta - Conjugatophyceae) em um lago de inundação amazônico de águas pretas (Lago Cutiuaú, Estado do Amazonas, Brasil). Acta Scientiarum. Biological Sciences 31(3): 235-243.

Monteiro, M.D.R.; Melo, N.F.A.C.; Alves, M.A.M.S. \& Paiva, R.S. 2009. Composition and distribution of the microphytoplankton from Guamá River between Belém and São Miguel do Guamá, Pará, Brazil. Boletim do Museu Paraense Emílio Goeldi. Ciências Naturais 4(3): 341-351.

Nogueira, M.G. 2000. Phytoplankton composition, dominance and abundance as indicators of environmental compartmentalization in Jurumirim Reservoir (Paranapanema River), São Paulo, Brazil. Hydrobiologia 431: 115-128.
Nogueira, M.G.; Ferrareze, M.; Moreira, M.L. \& Gouvêa, R.M. 2010. Phytoplankton assemblages in a reservoir cascade of a large tropical - subtropical river (SE, Brazil). Brazilian Journal of Biology 70(3): 781-793

Ohse, S.; Derner, R.B.; Ozório, R.A.; Braga, M.V.C.; Cunha, P.; Lamarca, C.P. \& Santos, M.E. 2009. Produção de biomassa e teores de carbono, hidrogênio, nitrogênio e proteína em microalgas. Ciência Rural 39(6): 1760-1767.

Oliveira, M.T.; Rocha, O. \& Peret, A.C. 2011. Structure of the phytoplankton community in the Cachoeira Dourada reservoir (GO/MG), Brazil. Brazilian Journal of Biology 71(3): 587-600.

Paiva, R.S; Eskinazi-leça, E; Passavante, J.Z.O; Silva-Cunha, M.G.G. \& Melo, N.F.A.C. 2006. Considerações ecológicas sobre o fitoplâncton da baía do Guajará e foz do rio Guamá (Pará-Brasil). Boletim do Museu Paraense Emílio Goeldi. Ciências naturais 1(2): 133-146.

Pereira, L.C.C.; Monteiro, M.C.; Guimarães, D.O.; Matos, J.B. \& Costa, R.M. 2010. Seasonal effects of wastewater to the water quality of the Caeté river estuary, Brazilian Amazon. Anais da Academia Brasileira de Ciências 82(2): 467-478.

Pereira, A.C.; Torgan, L.C. \& Melo, S. 2012. Pinnularia (Bacillariophyta) do curso inferior do rio Negro, Amazonas, Brasil: taxonomia e distribuição temporal. Acta Amazonica 42(3): 305-314.

Press, F.; Siever, R., Grotzinger, J., Jordan, T. H. 2003. Understanding Earth. 4 Ed. Bookman.

R Development Core Team. 2012. R: A language and environment for statistical computing R Foundation for Statistical Computing. Vienna. http://www.R-project.org

Raupp, S.V.; Torgan, L. \& Melo, S. 2009. Planktonic diatom composition and abundance in the Amazonian floodplain Cutiuaú Lake are driven by the flood pulse. Acta Limnologica Brasiliensia 21(2): 227-234.

Reynolds, C.S., Descy, J.P. \& Padisák, J. 1994. Are phytoplankton dynamic in rivers so different from those in shallow lakes? Hydrobiologia 285: 1-7.

Sala, S.E.; Duque, S.R.; Núñez-Avellaneda, M. \& Lamaro, A.A. 2002. Diatoms From the Colombian Amazon: Some Species of the Genus Eunotia (Bacillariophyceae). Acta Amazonica 32(4): 589-603.

Salmaso, N. \& Zygnin, A. 2010. At the extreme of physical gradients: phytoplankton in highly flushed, large rivers. Hydrobiologia 639: 21-36.

Scott, A.M., Grönblad, R.; Croasdale, H.T. 1965. Desmids from the Amazon Basin, Brazil, collected by Dr. H. Sioli. Acta Botannica Fennica 69: 3-93.

Silva, C.A.; Train, S. \& Rodrigues, L.C. 2001. Estrutura e dinâmica da comunidade fitoplanctônica a jusante e montante do reservatório de Corumbá, Caldas Novas, Estado de Goiás, Brasil. Acta Scientiarum 23(2): 283-290.

Soares, M.C.; Sophia, M.G. \& Huszar, V.L.M. 2007. Phytoplankton flora of two rivers in Southeast Brazil - Paraibuna and Pomba Rivers, Minas Gerais. Revista Brasileira de Botânica 30(3): 433-450.

Souza, K.F.; Melo, S. \& Almeida, F.F. 2007. Desmídias de um lago de inundação do Parque Nacional do Jaú (Amazonas-Brasil). Revista Brasileira de Biociências 5: 24-26

Souza, K.F. \& Melo, S.2011. Levantamento taxonômico de desmídias (Chlorophyta) do lago Novo (Amapá, Brasil): Gêneros Staurastrum, Staurodesmus e Xanthidium. Acta Amazonica 41(3): 335-346.

Sophia, M.G.; Huszar, V.L.M. 1996. Planktonic desmids of three Amazonian systems (Lake Batata, Lake Mussurá e Trombetas River), Pará, Brasil. Amazoniana 14: 75-90.

Thomasson, K. 1971. Amazonian algae. Mémoires de l'Institute Royale des Sciences Naturelles de Bélgique 10, 86: 1-57.

Townsed, C.R; Begon, M. \& Harper, J. L. 2008. Essentials of Ecology. Oxford. Blackwell Publishing.

Train, S.; Oliveria, M. D. \& Quevedo, M. T. 2000. Dinâmica sazonal da comunidade fitoplanctônica de um canal lateral (Canal Cortado) do Alto Rio Paraná (PR, Brasil). Acta Scientiarum 22(2): 389-395.

Train, S; Rodrigues, L.C. 1998. Temporal fluctuations of the phytoplankton community of the Baía River, in the upper Paraná River floodplain, Mato Grosso do Sul, Brazil, Hydrobiologia 361: 125-134.

Tucci, C.E.M. 1998. Modelo hidrológicos. Porto Alegre, Universidade / UFRGS/ Associação Brasileira de Recursos Hídricos. 
Tudesque, L.; Grenouillet, G.; Gevrey, M.; Khazraiec, K. \& Brosse, S. 2012. Influence of small-scale gold mining on French Guiana streams: Are diatom assemblages valid disturbance sensors? Ecological Indicators 14: 100- 106.

Wehr, J.D. \& Descy, J-P. 1998. Use of phytoplankton in large river management. Journal of Phycology 34: 741-749.
Wetzel, C.E.; Bicudo, D.C.; Ector, L.; Lobo, E.A. Soininen, J.; Landeiro, V.L. \& Bini, L.M. 2012. Distance Decay of Similarity in Neotropical Diatom Communities. PLOS ONE 7(9): 1-8.

Zeng, H.; Song, L.; Yu, Z. \& Chen, H. 2006. Distribution of phytoplankton in the Three-Gorge Reservoir during rainy and dry seasons. Science of the Total Environment 367: 999-1009. 